SLAC-PUB-8180

March 2000

Revision

\title{
Workshop Summary -- Accelerator Issues
}

\author{
Alex Chao
}

Stanford Linear Accelerator Center, Stanford University, Stanford, CA 94309

Work supported by Department of Energy contract DE-AC03-76SF00515. 


\title{
Workshop Summary - Accelerator Issues
}

\author{
Alex Chao \\ Stanford Linear Accelerator Center \\ P. O. Box 4349, Stanford, CA 94309, U.S.A.
}

\begin{abstract}
As we enter the high energy regime covered by RHIC and HERA, depolarization effects become strong, so that depolarization resonances begin to overlap. As a result, the "good old days" of the ZGS and AGS - when techniques for dealing with isolated resonances were sufficient - are now in the past, and a new generation of spin dynamics questions have to be addressed and new techniques have to be developed. Exciting results were presented at this workshop ranging from the recent rapid $\mathrm{R} \& \mathrm{D}$ advances on polarized $\mathrm{H}^{-}$sources to deeper understanding of the subtle spin dynamics involving Siberian snakes. This summary is an attempt to give some of the highlights.
\end{abstract}

\section{Introduction}

Problem with high energies The theme of the workshop is polarized protons at high energies. Unlike the orbital motions, spin motion is more sensitive at high energies. Roughly speaking, orbital perturbations in a storage ring are independent of beam energy $E$, while spin perturbations scale linearly with $E$. As a result, depolarization effects become stronger at higher energies. Two consequences follow:

(i) The depolarization resonances become wide and begin to overlap. Once this occurs, spin dynamics become very complicated and much harder to sludy. A new generation of spin simulation codes were created to address these problems, and we are increasingly dependent on these simulations.

(ii) To deal with the strong depolarization effects, Siberian snakes are necessary. These are ingenious devices, but they are also rich in subtleties and, indeed, surprises. Understanding them and making effective use of them has been a challenging occupation.

RHIC $\vec{p}$ and HERA $\vec{p}$ The two most noticeable polarized proton projects are HERA and RHIC. RHIC $\vec{p}$ is an approved project, aiming to provide polarized protons in 2001. HERA will make a decision about its polarized protons around 2003, and what we learn from RHIC by that time will be one of the inputs to the decision.[1] Table below is a comparison:

\begin{tabular}{|l|l|l|}
\hline RHIC & HERA & Equipments needed \\
\hline 25 GeV AGS & 8 GeV DESY-III & 1 partial snake, 1 RF dipole \\
$250 \mathrm{GeV}$ RHIC & 40 GeV PETRA & 2 full snakes \\
& $920 \mathrm{GeV}$ HERA & $\geq 4$ full snakes \\
\hline
\end{tabular}

From this table, it can be seen that, in the spin language, AGS is about equivalent to DESY-III, while RHIC is about equivalent to PETRA. 
Why is HERA $\vec{p}$ harder than RHIC $\vec{p}$ ? There are the following reasons:[2]

- HERA has a higher energy;

- RHIC has a planar layout, while three interaction regions of HERA contain vertical bends;

- HERA has only 4 straight sections to install Siberian snakes easily, while 8 would make things easier.

History A brief history of polarized protons was covered by Krisch at this workshop. [3] Table below gives another view:

\begin{tabular}{ll}
\hline Thomas-BMT equation & 1927,1959 \\
Froissart-Stora equation & 1960 \\
Sokolov-Ternov spontaneous polarization for electrons & 1964 \\
ZGS polarized beams & $1970 \mathrm{~s}$ \\
Siberian snake concept of Derbenev \&. Kondratenko & 1976 \\
AGS polarized beams - ZGS techniques & 1977 \\
AGS polarized beams - new techniques & 1994 - present \\
Snake experiments at IUCF & 1989 - present \\
HERA $\vec{e}$ longitudinal polarization reached $60 \%$ & 1994 \\
Spin proposals & \\
26 snakes SSC & 1993 \\
$\quad 6$ snakes Tevatron & 1994 \\
4 (8) snakes HERA & 1996 \\
2 snakes RHIC & 1997 \\
\hline
\end{tabular}

As seen from the table, we have gone a long way. In the 1970 s at the ZGS, we learned the techniques of using

- correction dipoles to overcome the imperfection $G \gamma=n$ resonances, and

- pulsed quadrupoles to overcome the intrinsic $G \gamma=n P \pm \nu_{y}$ resonances.

We successfully applied the same techniques to the AGS in 1977. More recently since 1994, a new generation of techniques have been applied to the AGS with exciting results. [4] More specifically, AGS now uses

- partial snake to overcome the imperfection resonances, and

- RF dipole to overcome the intrinsic resonances.

In the early ZGS and AGS days, depolarization resonances are relatively weak. They can be treated as isolated single resonances; Froissart-Stora equation are applicable and the beam dynamics are relatively simple. As mentioned earlier, the situation changes as we enter the energy regime of RHIC and beyond, when depolarization resonances begin to overlap.

\section{$2 \quad \mathrm{RHIC} \vec{p}$}

An overview of RHIC polarized proton project is given by Roser. [4] Highlights of recent studies include (i) the use of an RF dipole to spin flip the beam when crossing strong intrinsic resonances, and (ii) the use of a $5 \%$ solenoid partial snake to suppress the imperfection resonances. 
RF dipole Fig.1 shows the final beam polarization (after crossing the $G \gamma=12+\nu_{y}$ intrinsic resonance) as a function of the vertical coherent beam oscillation driven by the RF dipole. The spin flip is complete when the oscillation amplitude $\gtrsim 2 \sigma_{y}$.

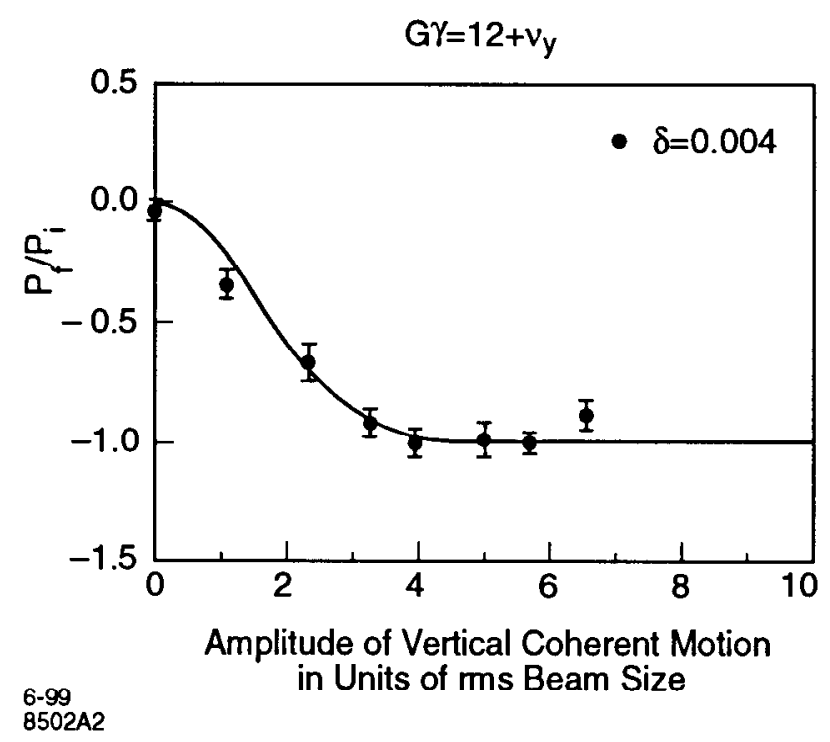

Figure 1: Spin flip by RF dipole in the AGS. $\delta$ is the difference between the RF tune and $\nu_{y}$.

Partial snake Fig.2 shows the beam polarization as a function of the final beam energy as a polarized beam is accelerated in the AGS, crossing depolarization resonances along the way. The two data points were obtained with the RF dipole on. The two curves were obtained by simulation.[5] The lower solid curve is to be compared with the measured data, and the agreement is rather good, indicating the simulation tool is doing its job. The reason the polarization is only $40 \%$ at the highest enegy is mostly due to the complication that the snake solenoid has the undesirable effect of coupling the $x-y$ orbital motions, which in turn excites the $G \gamma=n \pm \nu_{x}$ resonances. If the solenoid is replaced by a helical partial snake, as is planned, the simulated polarization is expected to look like the upper dashed curve.

Hybrid resonance In Fig.2, one notices a curious resonance at $G \gamma=60-9-\nu_{y}$. [6] This interesting resonance is a "hybrid" resonance driven by the product

$$
\text { (the 9-th harmonic of } \Delta x) \times\left(\epsilon_{y}\right)
$$

It can therefore be considered as a sideband of the intrinsic $G \gamma=60-\nu_{y}$ resonance, and can be compensated by adjusting the $\Delta x_{9}$ harmonics. The strength of this resonance is not of great concern, however.

Spin tracking Table below shows the strengths of the strongest depolarization resonance of various acclerators: 


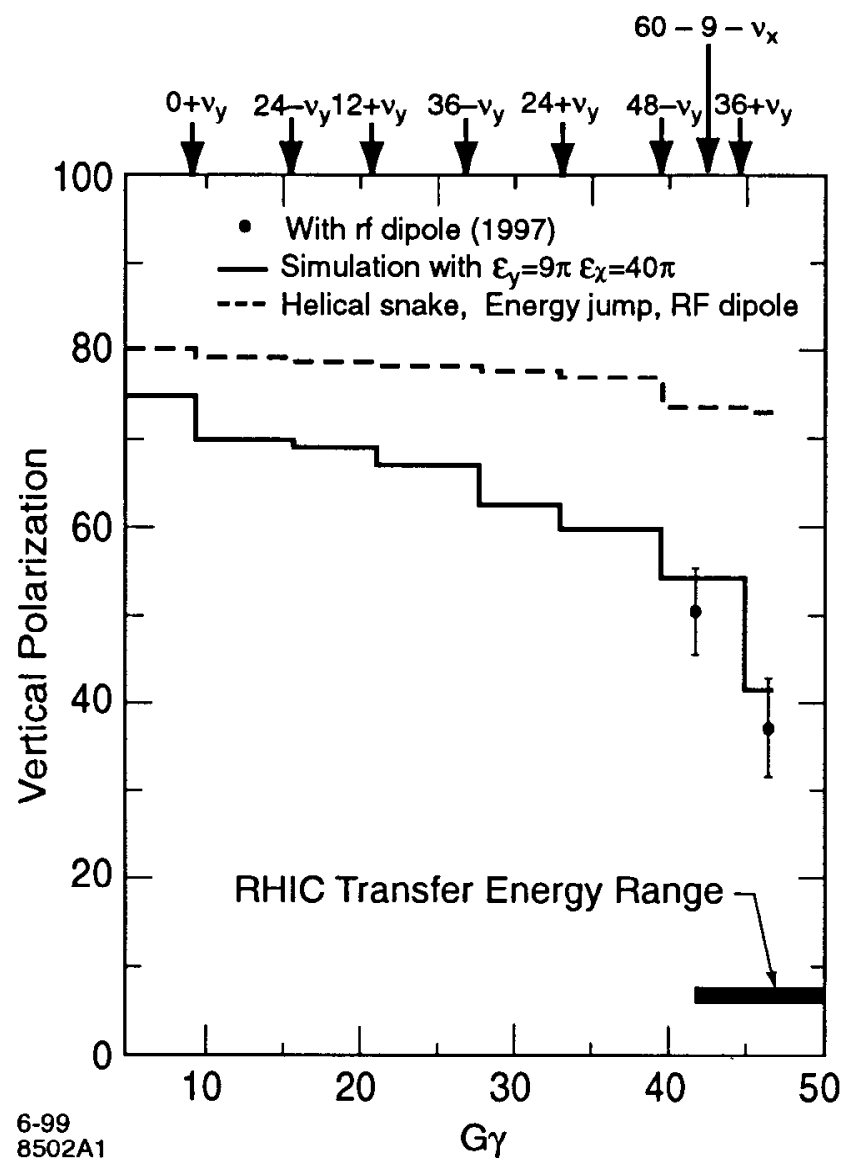

Figure 2: Beam polarization in the AGS with RF dipole and partial snake.

\begin{tabular}{lcc}
\hline & $E(\mathrm{GeV})$ & $\epsilon_{\max }$ \\
\hline AGS & 28 & 0.16 \\
RHIC $\vec{p}$ & 250 & 0.45 \\
DESY-III & 8 & 0.01 \\
PETRA & 40 & 0.06 \\
HERA $\vec{p}$ & 900 & 3.7 \\
\hline
\end{tabular}

It is seen that the maximum resonancc strength incrcases with the beam energy. As mentioned earlier, the stronger, and therefore overlapping (serious overlapping occurs when $\epsilon$ approaches 1), resonances are the reason that a new generation of spin tracking programs have been developed. For example, the program SPINK was developed for RHIC,[5] and the programs SPRINT [7, 2] and FORGET-ME-NOT[8] were developed for HERA.

Fig.3 shows one tracking result for RHIC (with 2 snakes) using SPINK. Assuming an rms vertical orbit distortion of $0.1 \mathrm{~mm}$, it is found that a particle at the beam edge (at $20 \pi \mathrm{mm}-\mathrm{mrad}$ ) would be completely depolarized by the time the beam is accelerated to $250 \mathrm{GeV}$. However, the beam as a whole basically has not sufferred too much loss of polarization. 


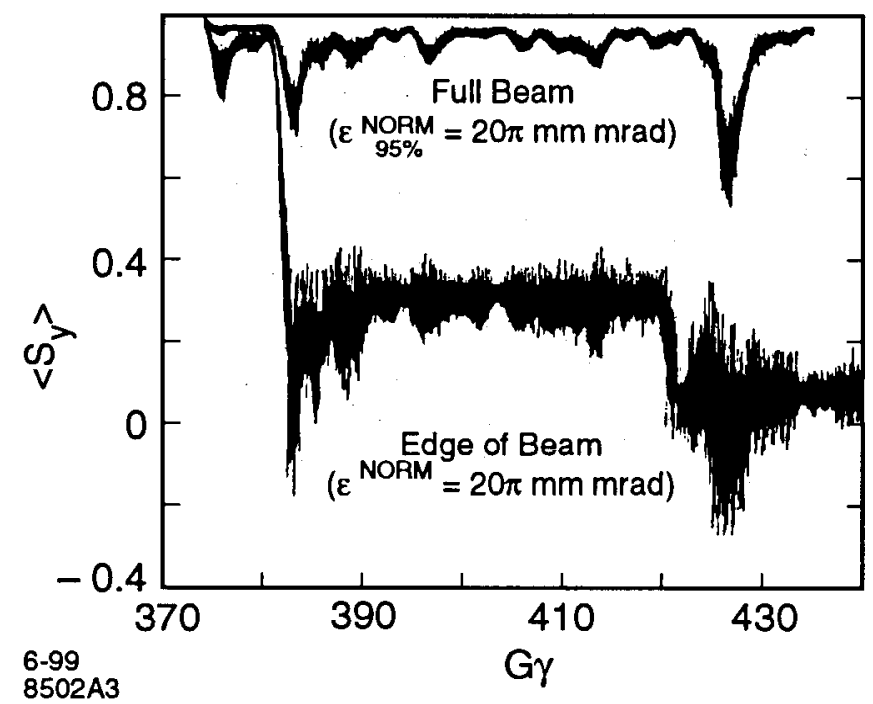

Figure 3: Expected polarization in RHIC by simulation.

\section{HERA $\vec{p}$}

An overview of the HERA polarized proton project was given by Hoffstätter. [2] It was pointed out [3] that there were two main technical issues facing HERA $\vec{p}$ : (i) bring the $\overrightarrow{\mathrm{H}}^{-}$source intensity to the needed level, and (ii) acceleration and storage of polarized proton beams in its chain of accelerators while keeping its polarization.

RFQ IHEP-Protvino has adopted an interesting RFQ design based on a 2-H structure instead of the more conventional 4-vane structure, as illustrated in Fig.4.[9] The advantage of the 2-H design is that it is expected to have a much larger beam acceptance of $\sim 2.4 \pi \mathrm{mm}$-mrad instead of the more common $\sim 1 \pi$ mm-mrad.
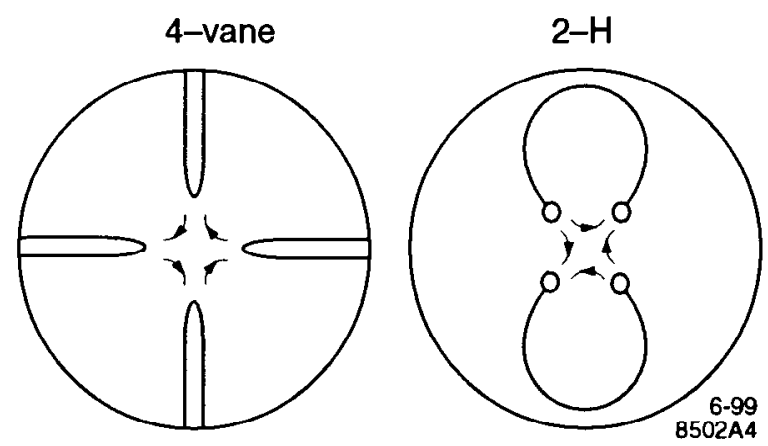

Figure 4: The 4-vane and the 2-H designs of RFQ.

Flattened snakes The problem of vertical bends in the interaction regions (IRs) is solved by the clever idea of "flattened snakes".[10] A flattened snake (FS) is a full Siberian snake with 
a radial spin rotation axis. An IR with vertical bend sections is sketched in Fig.5. There is one vertical bend section on each side of the interaction point. By inserting one FS at the middle of each of the vertical bend sections, the whole IR becomes spin transparent inspite of the vertical bends. The action of the two FSs cancel each other also, so there is no net snake action outside the IR (in the absence of errors). Several variants of the FS scheme are discussed in [12].

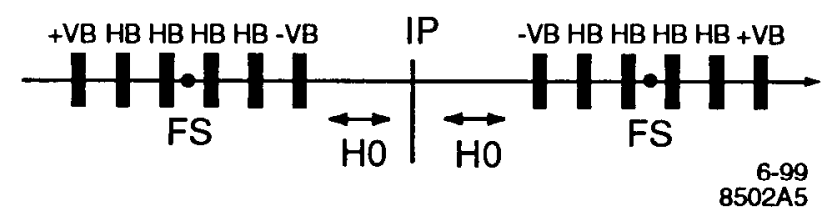

Figure 5: The scheme of flattened snakes.

Spin tracking Spin tracking has been carried out extensively for HERA. One result using FORGET-ME-NOT is shown in Fig.6.[8] The orbit distortions in this simulation were $\Delta x_{\mathrm{rms}}=$ $0.42 \mathrm{~mm}$ and $\Delta y_{\mathrm{rms}}=0.53 \mathrm{~mm}$, and the simulated beam has lost its polarization. It was concluded from these studies that (i) alignment errors play an important role, especially at high energies, and (ii) one can and should look for some special correction scheme to relax the alignment tolerances.

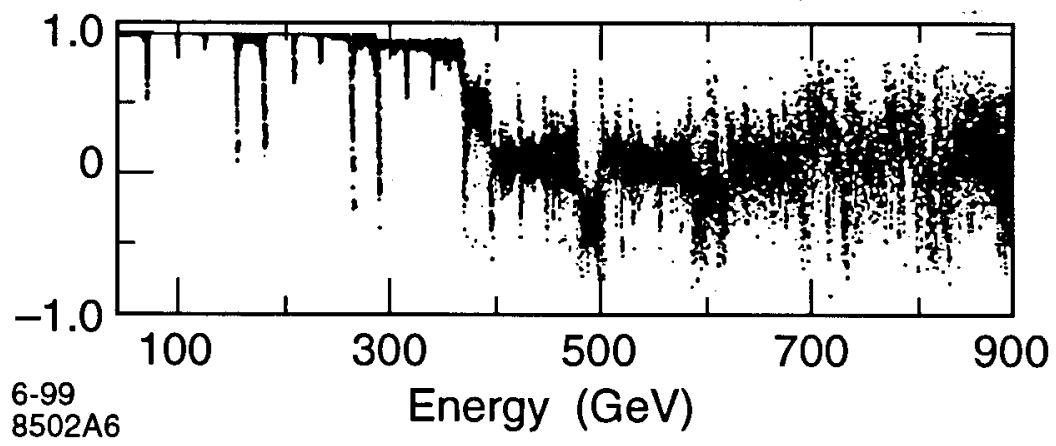

Figure 6: Simulation for HERA $\vec{p}$ using the program FORGET-ME-NOT.

A simulation using SPRINT is shown in Fig.7. [7] This simulation is for the case of 4 snakes, with a well-chosen $\nu_{y}$, and no misalignments. The upper figure shows that, by the time acceleration ends at $800 \mathrm{GeV}$, a good polarization is maintained for a particle with orbital amplitudes $\left(2 \sigma_{x}, 2 \sigma_{y}, 2 \sigma_{\delta}\right)$, while the lower figure says polarization is lost for a particle with amplitudes $\left(2.5 \sigma_{x}, 2.5 \sigma_{y}, 2.5 \sigma_{\delta}\right)$.

How many snakes for HERA? This is an important question for HERA. On the one hand, there is a strong preference to install 6 or 8 snakes to assure a good beam polarization, while on the other hand, there are only 4 straight sections where one find easy spaces for the snakes.

Unfortunately, theory is not loo clear on the criterion of the required number of snakes. One criterion by Lee and Courant[11] says the number of snakes, $N_{s}$, must be such that

$$
N_{s}>5 \epsilon_{\text {int }}, \quad N_{s}>10 \epsilon_{\text {imp }}
$$




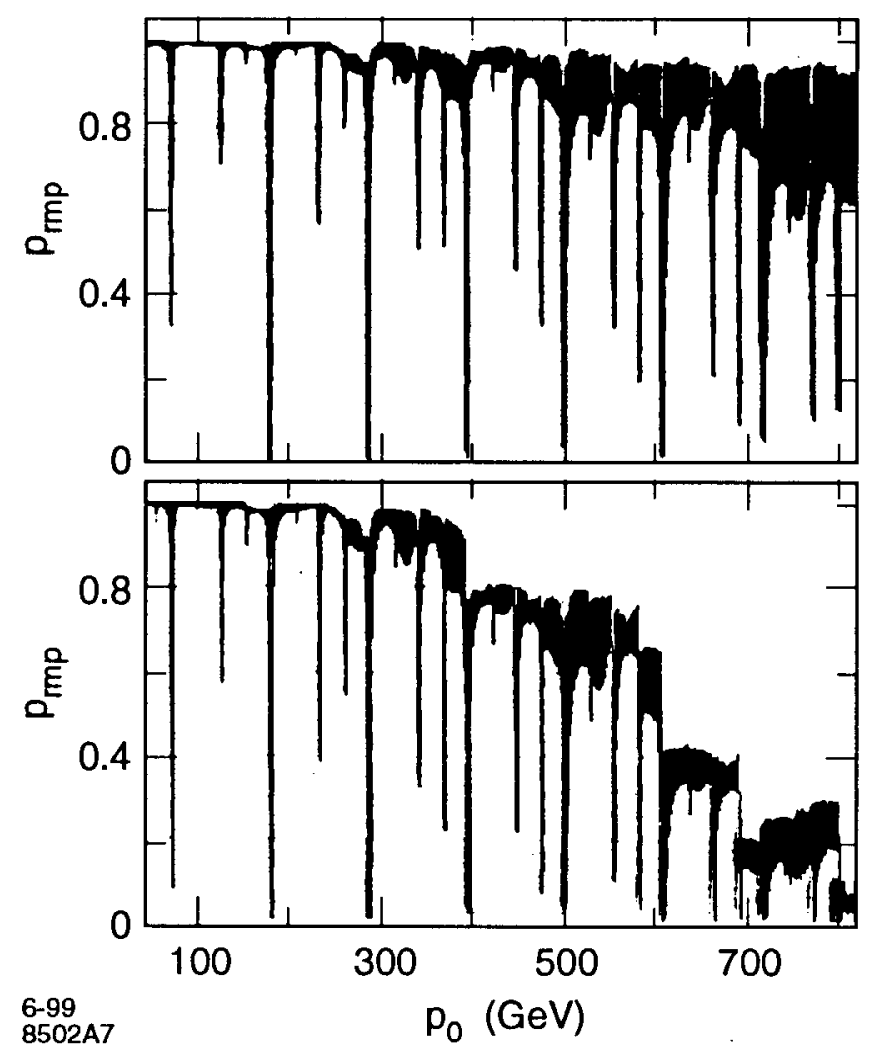

Figure 7: Simulation for HERA $\vec{p}$ using the program SPRIN'I'.

where $\epsilon_{\text {int,imp }}$ are the strengths for the strongest intrinsic and imperfection resonances. Another criterion by Anferov[10], however, suggestes

$$
N_{s}>4\left(\epsilon_{\mathrm{int}}+\epsilon_{\mathrm{imp}}\right)
$$

Fig. 8 shows the regions in the $\left(\Delta y_{\mathrm{rms}}, \epsilon_{N}\right)$ space allowed by these two criteria, where $\epsilon_{N}$ is the $95 \%$ normalized vertical beam emittance. (We have assumed $\epsilon_{\text {int }}=0.77$ with $5 \pi$ mm-mrad emittance and $\epsilon_{\mathrm{imp}}=0.36$ with $0.22 \mathrm{~mm}$ rms orbit.) The lower-left (shaded) region defined by each curve is the allowed region where beam polarization is expected to be maintained at the end of acceleration. One notes that the allowed regions are quite different using the two criteria, and very different if one has 4 or 8 snakes. Perhaps an effort of simulation could distinguish the validity of these two criteria.

Optimal 4-snake scheme Not all 4-snake schemes are equally effective. A snake is defined by its spin rotation axis. It was found empirically that the best performance was obtained by choosing the rotation axes $0^{\circ}, 45^{\circ}, 90^{\circ},-45^{\circ} .[7]$

One hypothesis suggested as the underlying principle for the best choice is as follows:[13] each snake should be "as perpendicular as possible" to its two neighbors. For 1 snakes, the best one can do is to have $45^{\circ}$ (not $90^{\circ}$ ) between adjacent snakes. 


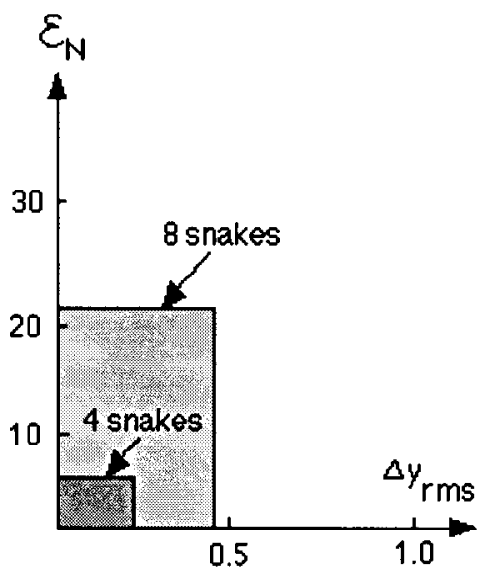

Lee-Courant criterion

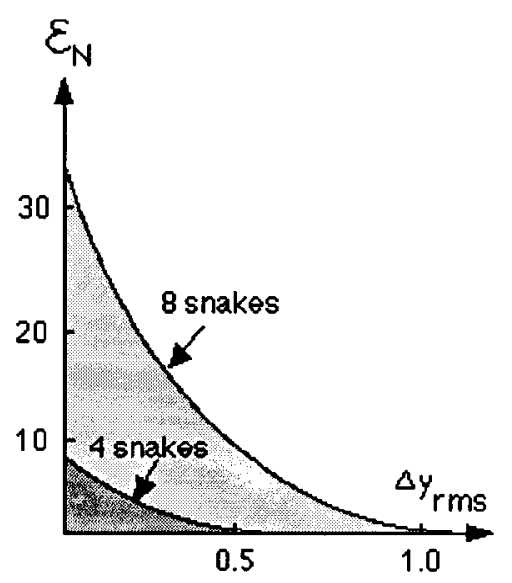

Anferov criterion

Figure 8: Two different existing snake criteria as applied to HERA $\vec{p}$. Which is correct?

Luminosity upgrade HERA is undergoing a luminosity upgrade.[14] The associated tighter orbit and emittance controls would help HERA $\vec{p}$ greatly. However, it is not clear whether these are sufficient to allow a 4 -snake HERA $\vec{p}$. In case it is not sufficient, considerations for HERA $\vec{p}$ include:

- further tightening on orbit and emittance,

- install 6 or 8 snakes by replacing some normal bends with snakes, and/or

- install electron cooling in PETRA at $15 \mathrm{GeV}$, which is expected to provide a cooling time of $3.5 \mathrm{~min}$. and a much smaller beam emittance.[15]

Beam-beam depolarization Beam-beam interaction is one potential mechanism for depolarization. 'T'here has been some beginning signs of that in HERA $\vec{e},[16]$ when it was observed that the electron beam polarization decreased with luminosity before September 1996, while it seems to become independent of the luminosity since then. The indication is that, at present, there might be some but not much depolarization for HER $\Lambda \vec{e}$. The qucstions rcmain as to (i) how about the protons, and (ii) what happens with the luminosity upgrade. These will require more studies.

As a rough guide, one might expect the beam-bcam depolarization to be specified by the parameter

$$
\sigma_{\theta}=(G \gamma+1) \frac{\sigma^{*}}{f}=(G \gamma+1) 4 \pi \xi \frac{\sigma^{*}}{\beta^{*}}
$$

where $\sigma^{*}$ is the rms beam size at the interaction point (IP), $f$ is the beam-beam focal length, $\xi$ is the beam-beam tune shift parameter, $\beta^{*}$ is the beta-function at the IP, and $\sigma_{\theta}$ is the rms spin rotation angle due to one beam-beam encounter. This model is consistent with a diffusion model of the orbital beam-beam interaction.

Table below gives the parameters[14, 17] for HERA $\vec{e}$ and HERA $\vec{p}$, before and after the luminosity upgrade. 


\begin{tabular}{|ll|c|c|}
\hline & & HERA 1999 & IIERA upgrade \\
\hline$\beta_{x}^{*}$ & $(\mathrm{~m})$ & $7(\mathrm{p}), 1(\mathrm{e})$ & $2.45(\mathrm{p}), 0.61(\mathrm{e})$ \\
$\beta_{y}^{*}$ & $(\mathrm{~m})$ & $0.5(\mathrm{p}), 0.7(\mathrm{e})$ & $0.18(\mathrm{p}), 0.26(\mathrm{e})$ \\
$\sigma_{x}^{*}$ & $(\mu \mathrm{m})$ & 205 & 118 \\
$\sigma_{y}^{*}$ & $(\mu \mathrm{m})$ & 55 & 32 \\
$\xi_{x}$ & & $0.001(\mathrm{p}), 0.025(\mathrm{c})$ & $0.0017(\mathrm{p}), 0.027(\mathrm{e})$ \\
$\xi_{y}$ & & $0.00035(\mathrm{p}), 0.01(\mathrm{e})$ & $0.00046(\mathrm{p}), 0.041(\mathrm{e})$ \\
$G \gamma$ & & $1758(\mathrm{p}), 68(\mathrm{e})$ & $1758(\mathrm{p}), 68(\mathrm{e})$ \\
\hline$\sigma_{\theta x}$ & $(\mathrm{mr})$ & $0.65(\mathrm{p}), 4.4(\mathrm{e})$ & $1.8(\mathrm{p}), 4.4(\mathrm{e})$ \\
$\sigma_{\theta y}$ & $(\mathrm{mr})$ & $0.85(\mathrm{p}), 0.68(\mathrm{e})$ & $1.8(\mathrm{p}), 4.4(\mathrm{e})$ \\
\hline
\end{tabular}

One sees that, for HERA $\vec{e}, \sigma_{\theta}$ stays at $4.4 \mathrm{mr}$ after the upgrade (at least in the $x$-direction), while for HERA $\vec{p}$, it increases from $0.65-0.85 \mathrm{mr}$ to $1.8 \mathrm{mr}$. If indeed $\sigma_{\theta}$ is a measure of beam-beam depolarization strength, the table indicates

- beam-beam depolarization is not much worsened with luminosity upgrade for HERA $\vec{e}$,

- but is about 3 times worsened for HERA $\vec{p}$.

Note that the tolerable value of $\sigma_{\theta}$ is not going to be the same for electrons and protons. The same reason explains why their beam-beam tune shift limits are so different. One expects a lower tolerance on $\sigma_{\theta}$ for protons than for electrons.

Beam-beam depolarization has been simulated for RHIC. A preliminary result is shown in Fig.9. [5] This simulation applies when the beams are colliding while accelerated. Various cases are for different initial particle displacements at the IP from 0.08 to $0.4 \mathrm{~mm}$.

\section{Helical Snakes}

Snake conditions There are 7 design conditions for a snake: 4 conditions to control orbits $\left(\Delta x=\Delta y=0, \Delta x^{\prime}=\Delta y^{\prime}=0\right)$, and 3 conditions for spin precession angle and axis.[10] In addition, there are desirable features imposed by (i) total length of the snake to be short, (ii) spin axis to be easily varied, and (iii) orbit deviation to be small inside the snake.

There are two types of snakes: one consisting of a series of horizontal and vertical bends, and the other consisting of a helical bend. In general, helical snakes are shorter and requires less $\int|B| d \ell$ than the upright bend type. A discussion of various helical snake designs can be found in [10].

RHIC helical snakes For RHIC, one first builds helical modules of the R and the $\mathrm{L}$ varieties, then the clever idea is that the same modules can be used for snakes and spin rotators, [18]

$$
\begin{aligned}
& \text { snake }=4 \text { modules RRRR } \\
& \text { spin rotator }=4 \text { modules RLRL }
\end{aligned}
$$

A prototype helical module has been built and cold tested. The magnet has reached the operating field of $4 \mathrm{~T}$ with a few quenches, and has reached the short sample limit of $5 \mathrm{~T}$. Dipole and sextupole field components have been mapped out through the manget length including the ends.[18] 

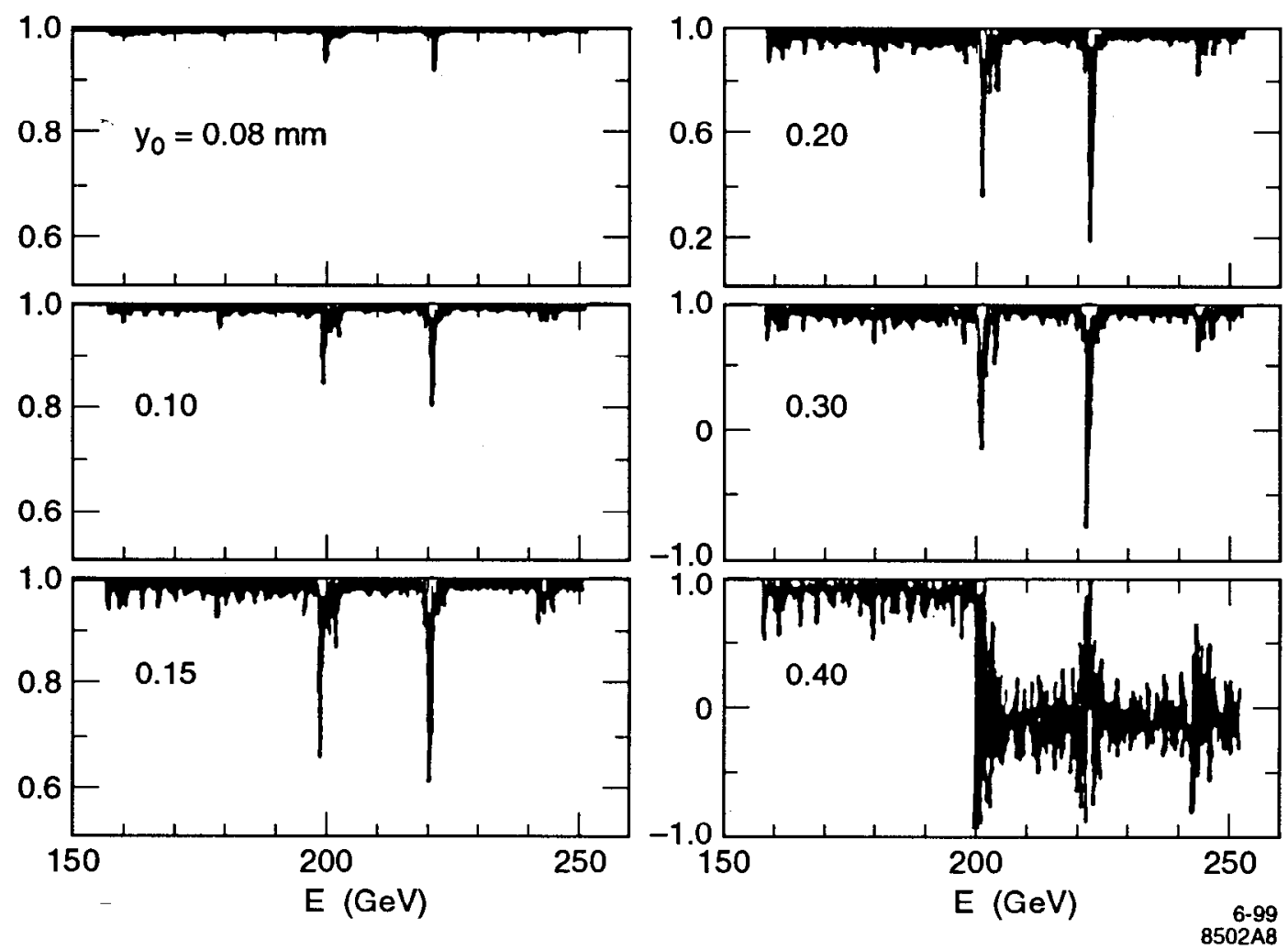

Figure 9: Beam-beam depolarization simulation for RHIC $\vec{p}$.

Effects on orbits As much as they are minimized, snakes have nonnegligible effects on particle's orbital motion. These have recently been studied using maps. A few such studies were discussed at the workshop.[19, 20,21] Such maps must be symplectic in its orbital description, and its spin map can be described using $\mathrm{SU}(2), \mathrm{O}(3)$, or quarternions. Helical snakes particularly have a large sextupole component (dictated by Maxwell equations). Compensation of these effects need some attention. It was pointed out that special attention is nceded for the ends of the helical magnets to make sure the magnet field is Maxwellian.[22]

\section{Polarized Sources}

Table below summarizes the present status of the sources in terms of what are needed and what have been achieved so far.

\begin{tabular}{|l|l|ccccc|}
\hline & & $\begin{array}{c}\text { polarization } \\
(\%)\end{array}$ & $\begin{array}{c}\text { intensity } \\
(\mathrm{mA})\end{array}$ & $\begin{array}{c}\text { pulse length } \\
(\mu \mathrm{s})\end{array}$ & $\begin{array}{c}\text { rep. rate } \\
(\mathrm{Hz})\end{array}$ & $\begin{array}{c}\text { emitt. } \\
(\mathrm{mm}-\mathrm{mrad})\end{array}$ \\
\hline \multirow{3}{*}{ Needed } & RHIC & & 0.5 & 300 & 7.5 & \\
& Tevatron & & 0.3 & 100 & 15 & $2 \pi$ \\
& HERA & 80 & 20 & 100 & 0.25 & $2 \pi$ \\
\hline \multirow{3}{*}{ Achieved } & KEK & 75 & 0.1 & 100 & 25 & $2 \pi$ \\
& Triumf/BINP & $55-60$ & 5 & 100 & $\mathrm{dc}$ & $0.3 \pi$ \\
& INR Moscow & 87 & 1 & 180 & 10 & $1.8 \pi$ \\
\hline
\end{tabular}


The specifications for the polarized HERA $\overrightarrow{\mathrm{H}}^{-}$source is very demanding and is not reached as yet. Its very high needed intensity of $20 \mathrm{~mA}$ results from the low repetition rate of $0.25 \mathrm{~Hz}$ limited by its injection system.

There are two types of $\overrightarrow{\mathrm{H}}^{-}$sources being considered for HERA, one is the more conventional atomic beam source (ABS), and the other is an optically pumped polarized ion source (OPPIS). Also discussed at the workshop were polarized deuteron[23] and helium sources[24].

ABS An ABS is being developed at INR, Moscow for HERA.[25] The scheme is illustrated in Fig.10.

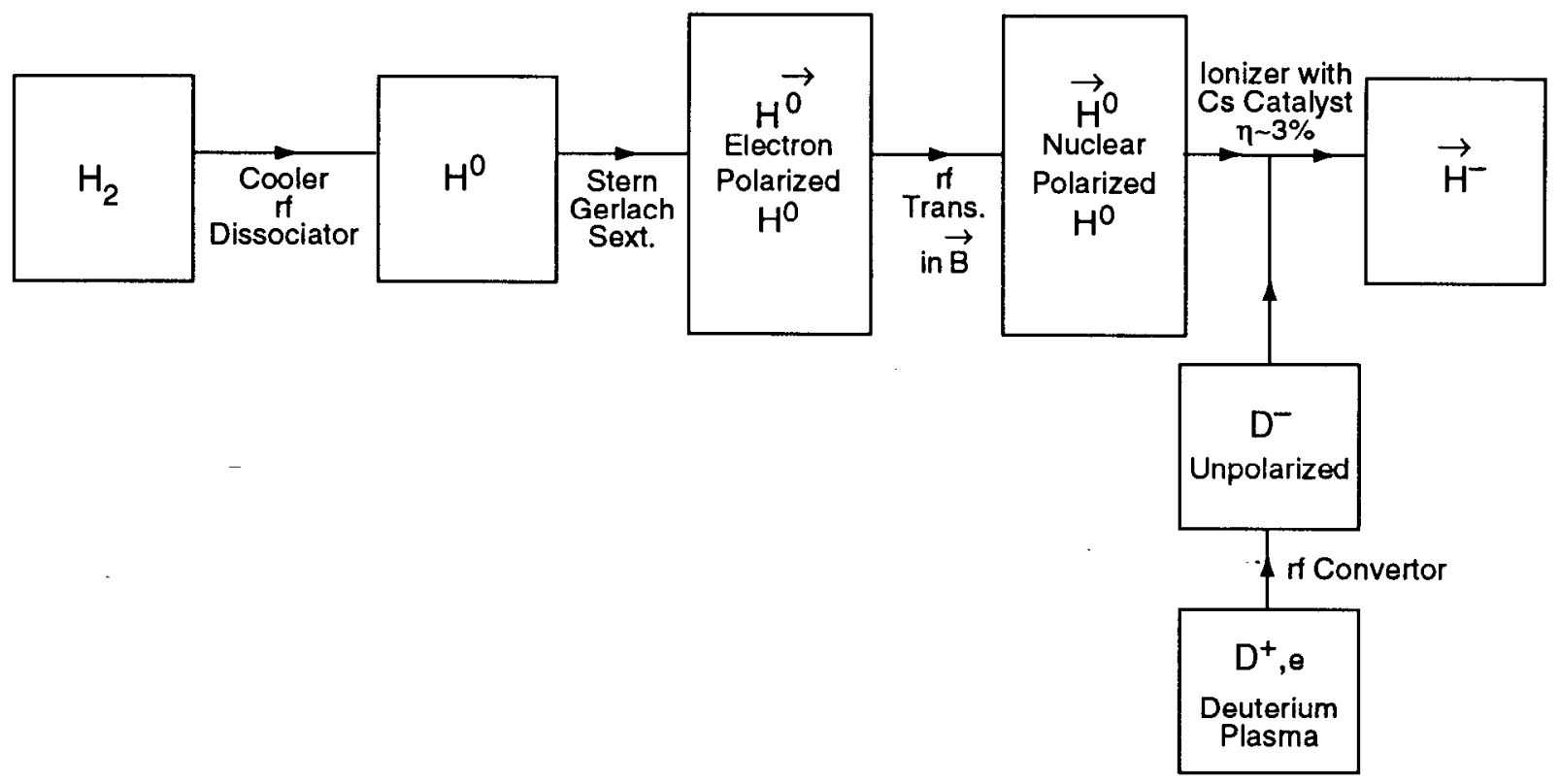

Figure 10: Schematics of an atomic beam source.

The presently achieved level of an ABS source has $1 \mathrm{~mA}$ beam with $87 \%$ polarization. The hope is to increase the beam intensity by a factor of 20 in half to one year time (provided funding).[25] The approach will consist of a combination of

- a more powerful $\mathrm{D}^{-}$source,

- higher $\mathrm{H}^{0}$ flux by a larger aperture sextupole, and

- installing a storage cell for $\overrightarrow{\mathrm{H}}^{-}$.

OPPIS An OPPIS is currently operating at RHIC.[4] It has basically reached its design goal and is ready for its expected operation in 2000. An OPPIS collaboration is being carried out by KEK, Triumf, and BINP. Schematics of an OPPIS is shown in Fig.11.|26, 27]

The presently achieved level of the OPPIS for HERA is $5 \mathrm{~mA}, 55-60 \%$ polarization. [27, 28] Higher beam intensity can be obtained by compromising on the polarization. It was guaranteed by the proponents that, in one year (with appropriate funding), the HERA specifications can be reached.[27] The steps to be taken include 


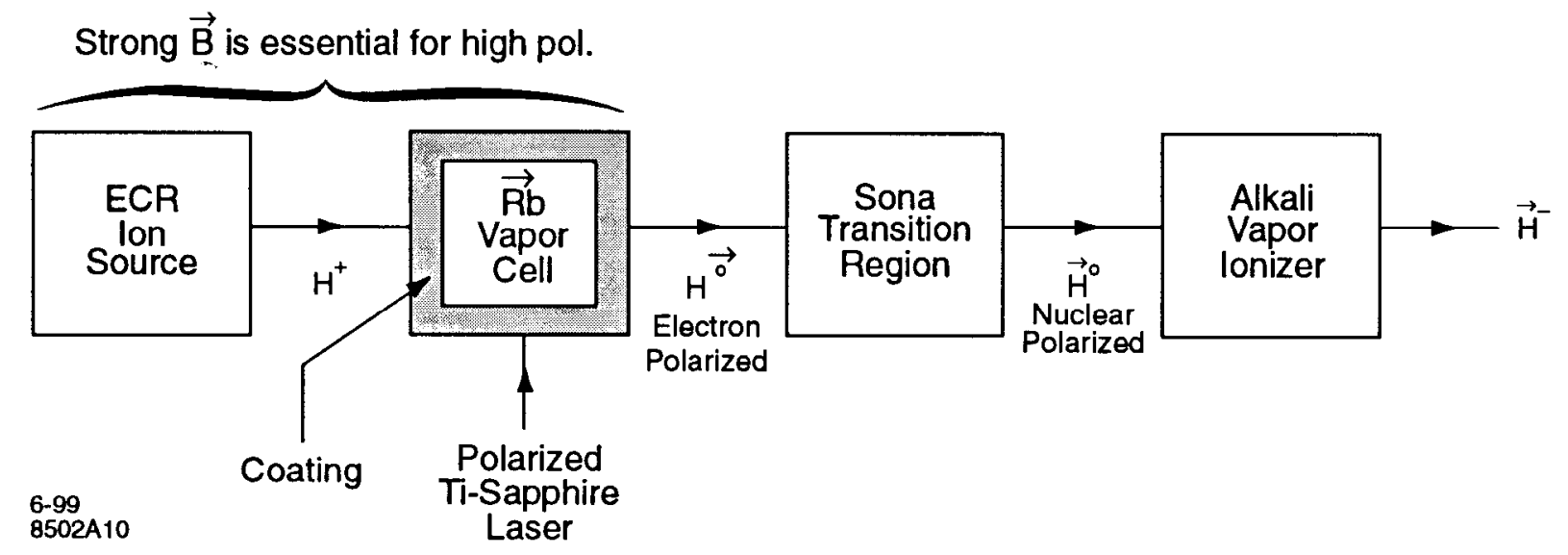

Figure 11: Schematics of an optically pumped polarized ion source.

- a spherical grid system for the $\mathrm{H}^{0}$ source,

- to replace the $10 \mathrm{kG}$ solenoid by a new superconducting $25 \mathrm{kG}$ solenoid, and

- replace the electron cyclotron resonance $\mathrm{H}^{+}$source by a BINP arc plasma $\mathrm{H}^{0}$ source.

Outlook Progress on polarized sources made in recent years has been very impressive. Development of both types of sources for HERA seems to be funding limited, and not technology limited. No "saturation effects" of all improvement ideas have been observed so far. Proponents of $\overrightarrow{\mathrm{H}}^{-}$sources are very optimistic in achieving the HERA specifications, provided appropriate funding. On the other hand, one must keep in mind that R\&D results are needed soon in order to provide input in time for the HERA $\vec{p}$ decision expected in 2003.

\section{Spin Dynamics}

The need to understand the spin dynamics in the era of strong (overlapping) resonances and with Siberian snakes has challenged the spin dynamists in the past decade. So far, the challenge is being met well, as evidenced by the new wave of insightful spin beam physics being developed, a good glimpse of which has been presented at this workshop. As mentioned, a new generation of spin tracking codes is one such effort. A few other efforts are mentioned below.

IUCF snake experiments A series of experiments on Siberian snakes is being performed at the IUCF sincc 1989.[3] In particular, an 1989 experiment demonstrated a snake overcomes imperfection resonances as expected, and an experiment in 1990 did the same for intrinsic resonances.

$\Lambda$ more recent IUCF experiment addressed the question of spin flip using an rf solenoid. When the Siberian snake was off, the spin flip efficiency was found to be about $99.9 \%$; however, when the snake was turned on, the efficiency dropped to about $97 \%$. 
One explanation of the reduced efficiency of spin flip due to the snake is as follows: [29] To induce a spin flip with snake on requires the rf solenoid to have a frequency $\nu_{\mathrm{rf}} \approx 1 / 2$. This frequency allows the possibility that some particles will not see the rf field at all if they happen to arrive the $\mathrm{rf}$ solenoid when the rf field is zero turn after turn.

The snake resonances Without Siberian snakes, the depolarization resonances are located at $\nu_{\text {spin }}=G \gamma=\left\{\begin{array}{l}n P \pm \nu_{y} \\ n\end{array}\right.$. Introduction of Siberian snakes does wonders to suppress these resonances, but however, do not eliminate them. These resonances re-appear as higher order (higher order in the resonance strength $\epsilon$ ) "snake resonances", which are located at[11]

$$
\nu_{\text {spin }}=\frac{1}{2}=m+(2 n+1) \nu_{y} \quad \Longrightarrow \quad \nu_{y}=\frac{2 m+1}{2(2 n+1)}
$$

\begin{tabular}{|c|c|c|c|c|c|c|}
\hline & $\mathrm{m}=0$ & $\mathrm{~m}=1$ & $\mathrm{~m}=2$ & $\mathrm{~m}=3$ & $\mathrm{~m}=4$ & $\begin{array}{c}\text { order in } \epsilon \\
=(2 n+1)\end{array}$ \\
\hline$n-0$ & $1 / 2$ & & & & & 1st order \\
\hline $\mathrm{n}=1$ & $1 / 6$ & $3 / 6$ & $5 / 6$ & & & $3 \mathrm{rd} \mathrm{ol}$ \\
\hline $\mathrm{n}=2$ & $1 / 10$ & $3 / 10$ & $5 / 10$ & $7 / 10$ & $9 / 10$ & 5 th order \\
\hline $\mathrm{n}=3$ & $1 / 14$ & $3 / 14$ & $5 / 14$ & $7 / 14$ & $9 / 14$ & 7 th order \\
\hline
\end{tabular}

Spin dynamics involving snakes tend to be subtle (keep in mind that these are intrinsically high order effects - except for the snake resonance at $\nu_{y}=1 / 2$ ). For example, theory says that even-ordered snake resonances are not driven unless resonances overlap, and are usually weak. Ironically, however, the first observed snake resonance (IUCF) was even-ordered, at $\nu_{y} \approx 3 / 4$.[10] The unexpected strength of this second-order snake resonance is not yet explained by theory or simulation.

A simulation has been performed for HERA to examine its expected snake resonance structure. Fig. 12 shows the result for the casc of a 4-snake HERA without orbit distortions.[30] One indeed observes the odd-ordered snake resonances. (Note that Fig.12 is plotted versus $\nu_{y}$ - not to be confused with the results previously shown, which were plotted versus the beam energy.) This result indicates that the snake resonances should not be a serious problem provided that (i) we choose $\nu_{y}$ carefully, (ii) the beam emittance is $10 \pi \mathrm{mm}$-mrad or less, and (iii) orbit distortion is sufficiently under control.

Spin invariant field The term "spin invariant field" has also been called "spin field" and "Derbenev-Kondrotenko vector" by the experts. It is the vector $\hat{n}(\vec{I}, \vec{\psi}, \theta)$ defined over the phase space $(\vec{I}, \vec{\psi})$, satisfying the Thomas-BMT equation, and the quasi-periodic condition

$$
\hat{n}(\vec{I}, \vec{\psi}+2 \vec{\pi}, \theta+2 \pi)=\hat{n}(\vec{I}, \vec{\psi}, \theta)
$$

Various nonperturbative (e.g. SPRINT) and perturbative (e.g. FORGET-ME-NOT, COSY) ways to calculate $\hat{n}$ have been invented and tested. It is reassuring that they all give the same correct result. $[2,7,21,31]$

Given this key quantity $\hat{n}$, one can then calculate the quantities

$$
\langle\hat{n}\rangle_{\text {phase space }} \text { and }\left\langle\hat{n} \cdot \hat{n}_{0}\right\rangle_{\text {phase space }}
$$

which give quick evaluation of the maximum achicvablc bcam polarization. This technique has been widely used in simulation studies as a pre-study before extensive tracking is performed. 


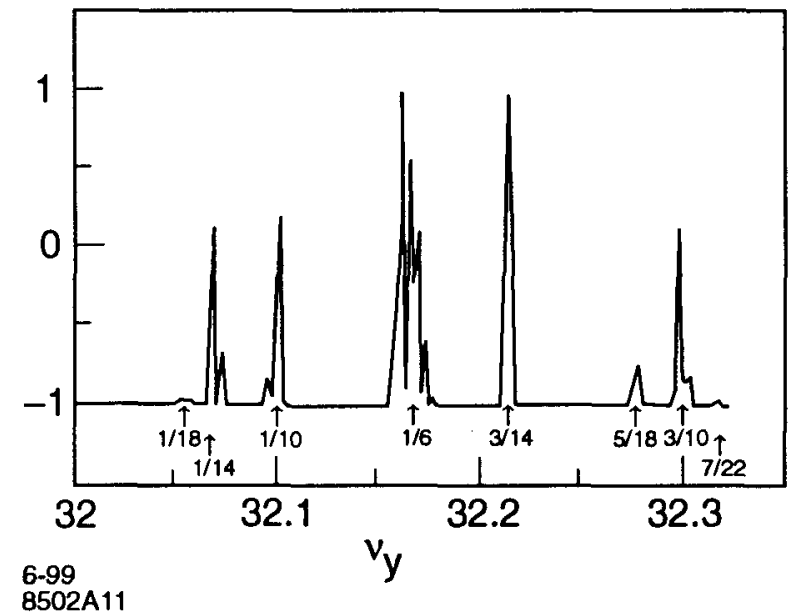

Figure 12: Simulation for HERA $\vec{p}$ for snake resonances.

Fokker-Planck approach A technique based on the Fokker-Planck diffusion equation has been developed.[32] Defining a spin density function $\overrightarrow{\mathcal{P}}(\vec{X}, s)$ in the phase space $(\vec{X})$, the diffusion equation looks like

$$
\begin{aligned}
\frac{\partial \overrightarrow{\mathcal{P}}}{\partial s}= & \hat{T}_{\text {Hamiltonian }} \overrightarrow{\mathcal{P}}+\vec{\Omega}_{\text {Thomas-BMT }}(\vec{X}, s) \times \overrightarrow{\mathcal{P}} \\
& +[\text { diffusion, damping, Sokolov-Ternov spin flip } \cdots]
\end{aligned}
$$

where $\hat{T}_{\text {Hamiltonian }}$ is the operator describing the orbital motion.

- Dropping the terms in the square brackets and imposing the stationary condition that $\overrightarrow{\mathcal{P}}$ is periodic in $s$ for all $\vec{X}$ give the spin invariant field $\hat{n}$.

- Beam polarization is given by $\overrightarrow{\mathcal{P}}_{\text {beam }}=\int \overrightarrow{\mathcal{P}} d^{6} X$.

- The depolarization time $\tau_{\text {depol }}$ can be obtained in terms of $\hat{n}, \partial \hat{n} / \partial \vec{X}, \partial^{2} \hat{n} / \partial \vec{X}^{2}$, etc. according to Eq.(7).

\section{References}

[1] A. Wagner, this workshop.

[2] G. Hoffstätter, this workshop.

[3] A. Krisch, this workshop.

[4] T. Roser, this workshop.

[5] A. Luccio, this workshop.

[6] T. Roser, M. Bai, this workshop.

[7] M. Vogt, this workshop. 
[8] N. Golubeva, V. Balandin, this workshop.

[9] J. Peters, this workshop.

[10] V.A. Anferov, this workshop.

[11] S.Y. Lee, E. Courant, Phys. Rev. D41, 292 (1990).

[12] Yu.M. Shatunov, this workshop.

[13] A. Krisch, communication.

[14] M. Seidel, this workshop.

[15] R. Brinkmann, this workshop.

[16] E. Gianfelice-Wendt, this workshop.

[17] F. Willeke, communication, this workshop.

[18] W. McKay, this workshop.

[19] C. Weissbäcker, this workshop.

[20] G. Wüstefeld, this workshop.

[21] V. Balandin, this workshop.

[22] A. Luccio, communication, this workshop.

[23] V. Fimushkin, this workshop.

[24] M. Romalis, this workshop.

[25] A. Belov, this workshop.

[26] L. Anderson, this workshop.

[27] A. Zelenski, this workshop.

[28] V. Davydenko, this workshop.

[29] S. Derbenev, communication, this workshop.

[30] E. Courant, communication.

[31] A. Lerach, this workshop.

[32] D. Barber, this workshop. 